\title{
HBX-related long non-coding RNA DBH-AS1 promotes cell proliferation and survival by activating MAPK signaling in hepatocellular carcinoma
}

\author{
Jin-lan Huang ${ }^{1, *}$, Ting-yu Ren ${ }^{1,}{ }^{*}$, Shun-wang Cao ${ }^{1}$, Shi-hao Zheng ${ }^{2}$, Xiu-mei Hu${ }^{1}$, \\ Yan-wei Hu시 Li Lin ${ }^{1}$, Jing Chen ${ }^{1}$, Lei Zheng ${ }^{1}$ and Qian Wang ${ }^{1}$ \\ ${ }^{1}$ Laboratory Medicine Center, Nanfang Hospital, Southern Medical University, Guangzhou, Guangdong, China \\ 2 Department of Neurosurgery, Fujian Provincial Hospital, Fuzhou, Fujian, China \\ "These authors have contributed equally to this work \\ Correspondence to: Qian Wang, email: wangqian@smu.edu.cn
}

Lei Zheng, email: nfyyzhenglei@smu.edu.cn

Keywords: IncRNA, DBH-AS1, HCC, proliferation, HBx

Received: March 04, $2015 \quad$ Accepted: August 23, 2015

Published: September 15, 2015

This is an open-access article distributed under the terms of the Creative Commons Attribution License, which permits unrestricted use, distribution, and reproduction in any medium, provided the original author and source are credited.

\section{ABSTRACT}

Accumulating evidence supports an important role for the hepatitis $B$ virus $x$ protein (HBX) in the pathogenesis of hepatitis B virus (HBV)-induced hepatocellular carcinoma (HCC), but the underlying mechanisms are not entirely clear. Here, we identified a novel long noncoding RNA (IncRNA) DBH-AS1 involved in the HBXmediated hepatocarcinogenesis. The levels of DBH-AS1 were positively correlated with hepatitis B surface antigen (HBsAg) and tumor size in HCC tissues. Functionally, transgenic expression of DBH-AS1 significantly enhanced cell proliferation and tumorigenesis, whereas short hairpin RNA knockdown of DBH-AS1 caused an inhibition of cell proliferation. Mechanistically, overexpression of DBH-AS1 induced cell cycle progression by accelerating $\mathrm{G1} / \mathrm{S}$ and $\mathrm{G2} / \mathrm{M}$ transition concomitantly with upregulation of CDK6, CCND1, CCNE1 and downregulation of p16, p21 and p27. We also found that enhanced DBH-AS1 expression inhibited serum starvation-induced apoptosis of HCC cells. In contrast, suppressed DBH-AS1 expression had opposite effects. Furthermore, DBH-AS1 was shown to activate MAPK pathway. We also provide evidence that DBH-AS1 could be significantly induced by HBx protein and markedly down-regulated by p53. Thus, we concluded that DBH-AS1 can be induced by HBX and inactivated by $\mathrm{p} 53$, and consequently promote cell proliferation and cell survival through activation of MAPK signaling in HCC. Our study suggests that DBH-AS1 acts as an oncogene for HCC.

\section{INTRODUCTION}

Hepatocellular carcinoma (HCC), accounting for $90 \%$ of primary liver cancers, is a highly lethal cancer with increasing worldwide incidence [1]. There are more than 250000 new HCC cases and estimated 500 000-600 000 deaths due to this disease annually [1-3]. Despite of the therapeutic advances that had been made in HCC in the past few decades, such as surgical resection, liver transplantation, and adjuvant therapy, the overall 5-year survival rate of HCC patients still remains poor [4]. The major risk factor for $\mathrm{HCC}$ is chronic infection with hepatitis B virus (HBV), which accounts for $52 \%$ of all HCC [5]. HBV X protein (HBx), encoded by HBV x gene, has been implicated to act as a multifunctional oncogenic factor in the development of HBV-related HCC, including promoting cell cycle progression, inactivating negative growth regulators, regulating apoptosis and inhibiting nucleotide excision repair of damaged cellular DNA [6]. However, the molecular mechanisms underlying HBx protein-mediated tumorigenesis are not entirely clear.

Long non-coding RNAs (lncRNAs) are defined as transcribed RNA molecules greater than 200nt in length with limited or no protein-coding capacity [7]. Recent 
studies have demonstrated that various lncRNAs, such as HULC, ATB, HOTAIR, HOTTIP, URHC, Dreh, UFC1, can participate in diverse biological processes involved in hepatocarcinogenesis, including cell proliferation, apoptosis and metastasis [8-11]. Importantly, several lncRNAs have been identified to be related to $\mathrm{HBx}$ protein. Huang, J. F. et al [12] characterized a lncRNA Dreh in mouse which could be down-regulated by HBx protein. Dreh was elucidated to inhibit HCC metastasis by targeting the intermediate filament protein vimentin. Furthermore, HULC, a highly expressed lncRNA in HCC, was found to be elevated by HBx protein and promote hepatocyte proliferation via down-regulating p18 [13]. Although several lncRNAs have been demonstrated to be regulated by $\mathrm{HBx}$ protein, the specific role of $\mathrm{HBx}$-related lncRNAs in HCC remains largely unknown.

Recently, a microarray analysis carried out by Magkoufopoulou, C. et al [14] revealed that quercetin results in decreased expression of IncRNA DBH-AS1 (NCBI Accession NO. NR_102735; UCSC ID uc031tfk.1) in HepG2 cells. DBH-AS1 is a $\sim 2 \mathrm{~kb} \operatorname{lncRNA}$ with a polyadenylated tail transcribed from chromosome 9q34 [15]. Quercetin, the prototype of a naturally-occurring chemopreventive agent for various cancers, has been reported to possess anti-proliferative and antioxidant activities in hepatocytes [16-18]. Hsieh, A. et al [19] found that quercetin effectively represses $\mathrm{HBx}$-mediated regulation of several key oncogenes. However, whether DBH-AS1 is involved in the development of HBV-related HCC has not been elucidated so far.

In this study, we first assessed the levels of DBHAS1 in 45 HCC tumor tissues. The clinical data of the patients in our study showed that high levels of DBH-AS1 are positively associated with tumor size and hepatitis B surface antigen (HBsAg). Further investigation of the function of DBH-AS1 in HCC revealed that DBHAS1 promotes cell proliferation and survival. Moreover, activation of the ERK/p38/JNK MAPK signaling pathway is observed in cells overexpressing DBH-AS1. Furthermore, DBH-AS1 is found to be significantly induced by HBx protein and inactivated by p53. Totally, these results suggested that DBH-AS1 exerts an impact as a potential oncogene and may provide us new insight into the role of HBx-related lncRNAs in the development of HCC.

\section{RESULTS}

\section{Associations between IncRNA DBH-AS1 expression and clinicopathological characteristics}

To investigate the role of DBH-AS1 in $\mathrm{HCC}$, we examined DBH-AS1 expression in $45 \mathrm{HCC}$ tumor tissues by qRT-PCR. Statistical analysis revealed that high DBH-
AS1 levels were positively correlated with tumor size $(\chi 2=8.006, P=0.005)$ and HBsAg $(\chi 2=4.132, P=$ 0.042 , Table 1$)$. However, we did not find any correlation between DBH-AS1 levels and other clinicopathological features, including gender, age, AFP level, liver cirrhosis, tumor number and Edmondson grade. These data indicate that DBH-AS1 may be involved in HCC tumor growth and potentially be related to $\mathrm{HBV}$ infection.

\section{LncRNA DBH-AS1 promotes HCC cell proliferation in vitro}

To evaluate the biological functions of $\mathrm{DBH}-$ AS1, the levels of DBH-AS1 in HCC cell lines and hepatic immortal cell lines were analyzed by qRT-PCR. Higher levels of DBH-AS1 were observed in the SKHep1, Hep3B, MHCC97H and HepG2 cell lines than in the hepatic immortal cell line LO2 and QSG7701 (Supplementary Figure S1A).

To further investigate the biological effect of DBH-AS1 in HCC cells, we constructed HepG2 and SMMC-7721 cell lines with stably overexpressed DBHAS1, and Hep3B and SK-Hep1 cell lines with stably silenced DBH-AS1 expression (Figure 1A). The growth curves determined by CCK-8 assays indicated that cell proliferation was enhanced by overexpression of DBH-AS1 in HepG2 and SMMC-7721 cells, whereas knockdown of endogenous DBH-AS1 expression dramatically reduced the proliferative capacity of Hep3B and SK-Hep1 cells (Figure 1B). Further colony formation assays also showed that up-regulation of DBH-AS1 could significantly enhance the colony formation ability in HepG2 and SMMC-7721 cells. In contrast, suppressed DBH-AS1 expression had the opposite effect (Figure 1C). Thus, these data revealed that DBH-AS1 enhances HCC cell growth.

\section{LncRNA DBH-AS1 promotes tumor growth in vivo}

To determine the growth-enhancing effect of DBH-AS1 in vivo, we injected SMMC-7721 cells stably transfected with Lv-DBH-AS1 or Lv-control subcutaneously into nude mice for xenotransplantation. Mice injected with cells overexpressing DBH-AS1 showed significantly increased tumor growth compared to those injected with cells transfected with LV-control (Figure 2A). As assessed by measurements of tumor volume and mass, enhanced DBH-AS1 expression significantly promoted overall tumor growth (Figure 2B2D). The immunohistochemistry analysis of the tumor tissues from xenografts revealed that the expression of Ki67 proliferation antigen was significantly stronger in xenografts of Lv-DBH-AS1 cells than in xenografts of Lv-control cells (Figure 2E). These results showed that 
Table 1: Correlation between IncRNA DBH-AS1 expression and clinicopathological characteristics in HCC patients $(n=45)$.

\begin{tabular}{|c|c|c|c|c|}
\hline \multirow[t]{2}{*}{ Variables } & \multicolumn{2}{|c|}{ IncRNA DBH-AS1 expression } & \multirow[t]{2}{*}{$\mathbf{x} 2$} & \multirow[t]{2}{*}{ "P Value } \\
\hline & Low & $\operatorname{High}^{\#}$ & & \\
\hline All cases & 22 & 23 & & \\
\hline Age & & & 0.218 & 0.641 \\
\hline$>55$ & 9 & 11 & & \\
\hline$<=55$ & 13 & 12 & & \\
\hline Gender & & & 0.505 & 0.477 \\
\hline Male & 19 & 18 & & \\
\hline Female & 3 & 5 & & \\
\hline HBsAg & & & 4.132 & $0.042^{*}$ \\
\hline positive & 12 & 19 & & \\
\hline negative & 10 & 4 & & \\
\hline Liver cirrhosis & & & 1.779 & 0.182 \\
\hline with & 10 & 15 & & \\
\hline without & 12 & 8 & & \\
\hline $\operatorname{AFP}(\mathbf{n g} / \mathrm{ml})$ & & & 0.192 & 0.661 \\
\hline$>\mathbf{2 0}$ & 11 & 10 & & \\
\hline$<=\mathbf{2 0}$ & 11 & 13 & & \\
\hline Tumor size(cm) & & & 8.006 & $0.005^{* *}$ \\
\hline$>5 \mathrm{~cm}$ & 7 & 17 & & \\
\hline$<=5 \mathrm{~cm}$ & 15 & 6 & & \\
\hline Tumor number & & & 0.505 & 0.477 \\
\hline solitary & 19 & 18 & & \\
\hline multiple & 3 & 5 & & \\
\hline Edmondson grade & & & 0.67 & 0.413 \\
\hline I+II & 2 & 4 & & \\
\hline III & 20 & 19 & & \\
\hline
\end{tabular}

\footnotetext{
"The median expression level was used as the cutoff. Low expression of lncRNA DBH-AS1 in 22 patients was classified as values below the 50th percentile. High lncRNA DBH-AS1 expression in 23 patients was classified as values at or above the 50th percentile.

*For analysis of correlation between IncRNA DBH-AS1 levels and clinical features,Pearson's chi-square tests were used. $*, P<0.05 ;{ }^{* *}, P<0.01$.
} 
DBH-AS1 accelerates HCC cell proliferation in vivo.

\section{LncRNA DBH-AS1 induces cell-cycle progression in $\mathrm{HCC}$ cells}

To gain insights into the mechanism by which DBHAS1 enhances HCC cell proliferation, EdU incorporation assays and fluorescence-activated cell sorting (FACS) were performed to analyze differences in cell-cycle distributions after DBH-AS1 overexpression or silencing. EdU incorporation assays showed that overexpression of DBH-AS1 significantly increased the percentage of EdU positive cells in HepG2 and SMMC-7721 cells whereas DBH-AS1 depletion resulted in a marked reduction in the percentage of EdU positive cells in Hep3B and SKHep1 cells, indicating that DBH-AS1 facilitates the entry of cells into S phase (Figure 3A). Consistent with our EdU results, a reduction in the G1 population and an increase in the $\mathrm{S}$ and $\mathrm{G} 2 / \mathrm{M}$ population were observed in HepG2 and SMMC-7721 cells overexpressing DBH-AS1. Conversely, repressed DBH-AS1 expression in Hep3B and SK-Hep1 cells mainly led to a G1 accumulation and a decrease of $\mathrm{S}$ and $\mathrm{G} 2 / \mathrm{M}$ phase (Figure $3 \mathrm{~B}$ and $3 \mathrm{C}$ ). Furthermore, we also examined the levels of several key genes involved in cell cycle checkpoint in HepG2 cells stably overexpressing DBH-AS1 and Hep3B cells with silenced DBH-AS1 expression by qRT-PCR and western blot analysis. Overexpression of DBH-AS1 in HepG2 cells elevated the expression of oncogenic cell-cycle regulators including CDK6, CCND1, CCNE1, but reduced the expression of cyclin-dependent protein kinase inhibitors including p16, p21, p27 (Figure 3D and 3E). By contrast, knockdown of DBH-AS1 in Hep3B cells resulted in a decreased expression of CDK6, CCND1, CCNE1 and an increased expression of p16, p21, p27 (Figure 3D and 3E).

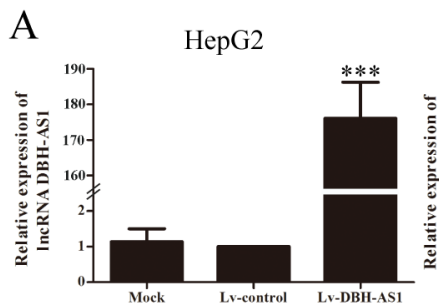

$\mathrm{C}$

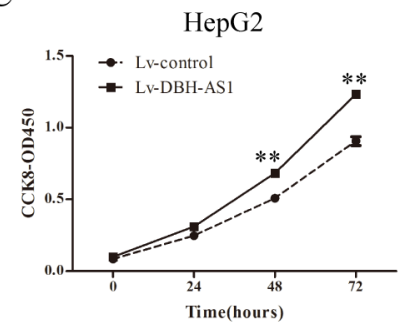

$\mathrm{E}$

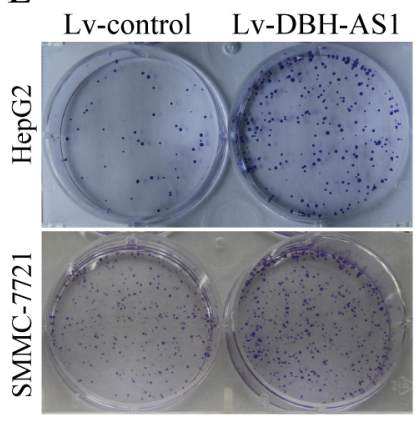

SMMC-7721
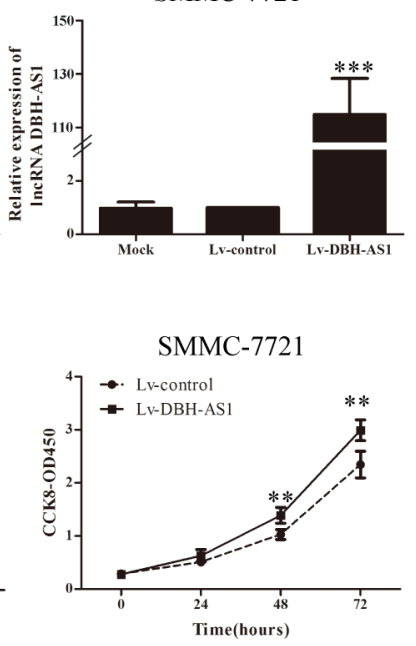

D

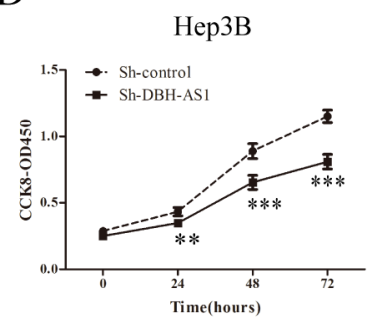

F

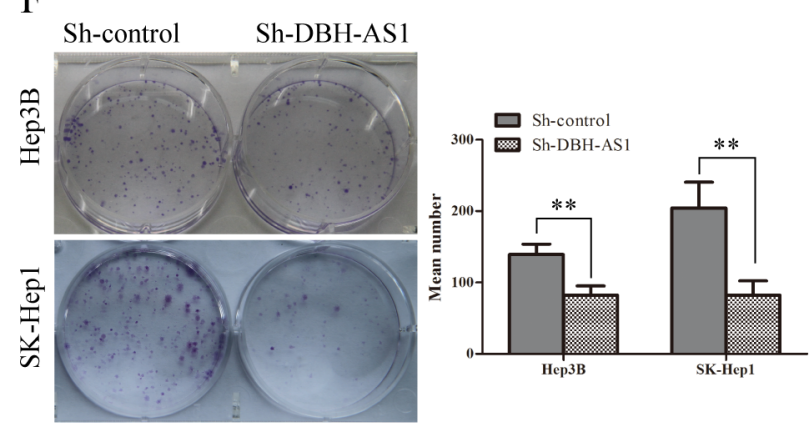

Figure 1: LncRNA DBH-AS1 promotes HCC cell proliferation in vitro. A. HepG2 and SMMC-7721 cells were infected with lentivirus carrying the DBH-AS1 gene, and HepG2 and SMMC-7721 cells stably overexpressing DBH-AS1 were screened by qRT-PCR. B. Short hairpin RNA against DBH-AS1 stably decreased the expression of DBH-AS1 in sh-DBH-AS1 Hep3B and SK-Hep1 cells compared with sh-control cells by qRT-PCR. C. After overexpression of DBH-AS1 in HepG2 and SMMC-7721 cells, the cell viability was assessed by CCK-8 assays daily for 3 days. D. Cell viability was assessed by CCK-8 assays daily for 3 days in Hep3B and SK-Hep1 cells with silenced DBH-AS1 expression. E. Colony formation assays were performed on HepG2 and SMMC-7721 cells stably overexpressing DBHAS1 for 2 weeks. F. In vitro proliferative ability of Hep3B and SK-Hep1 cells was significantly decreased in DBH-AS1-suppressed cells compared to sh-control cells by colony formation assays. Data are presented as mean $\pm \mathrm{SD}$ for at least three independent experiments, $* P$ $<0.05, * * P<0.01, * * * P<0.001$. 


\section{LncRNA DBH-AS1 inhibits serum starvation- induced apoptosis of $\mathrm{HCC}$ cells}

Because DBH-AS1 exerts an oncogenic effect in HCC cells, we speculated that DBH-AS1 may be critical for cell survival and apoptosis. To test this hypothesis, we further checked the effect of DBH-AS1 on HCC cell survival. Apoptosis was measured by FACS-based Annexin-V/7-AAD double staining in HCC cells under serum starvation condition for $48 \mathrm{~h}$. The results revealed that the percentage of Annexin V-positive cells was lower in DBH-AS1-overexpressing HepG2 and SMMC-7721 cells than control cells. In contrast, sh-DBH-AS1 Hep3B and SK-Hep1 cells had a significantly higher percentage of Annexin V-positive cells than sh-con group (Figure 4A and 4B). We further examined the expression of 2 welldefined apoptosis protein markers, caspase 3 and its active form of cleaved caspase 3 . The ratio of cleaved caspase 3/caspase 3 was remarkably decreased in HepG2 cells with elevated DBH-AS1 expression, whereas the opposite effects could be found in Hep3B cells with suppressed DBH-AS1 expression (Figure 4C). Collectively, our data suggest that DBH-AS1 inhibits serum starvation-induced apoptosis and enhances HCC cell survival.

\section{LncRNA DBH-AS1 activate MAPK signaling pathways in HCC cells}

Mitogen-activated protein kinases (MAPKs), including extracellular signal-regulated kinase (ERK),
A

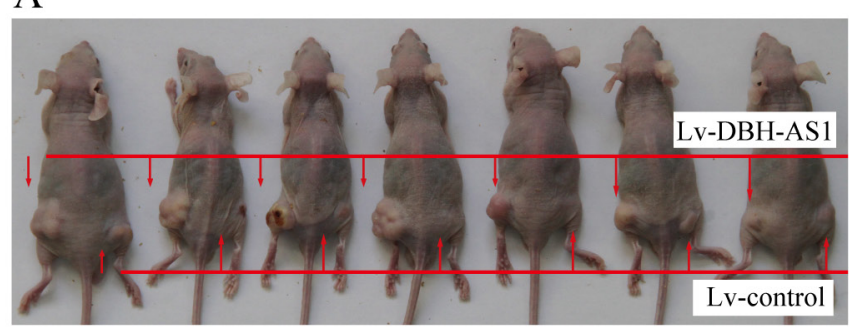

$\mathrm{C}$

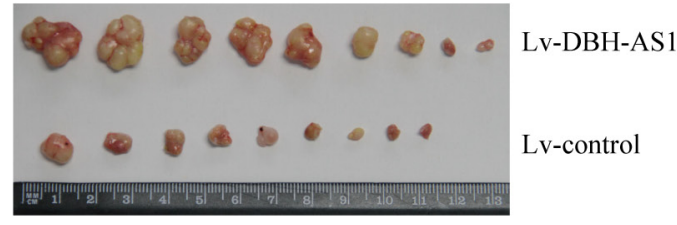

E

Lv-control

Lv-DBH-AS1

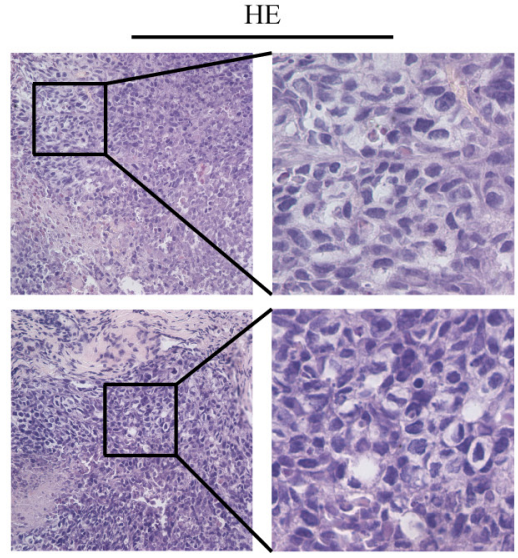

B

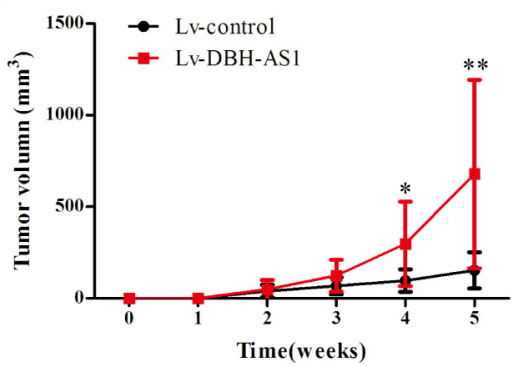

$\mathrm{D}$
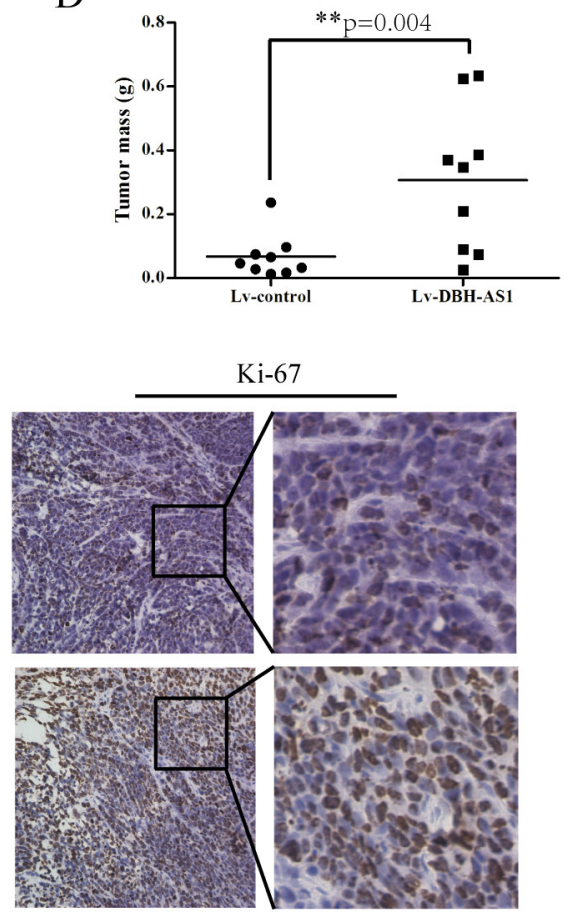

Figure 2: LncRNA DBH-AS1 accelerates tumor growth in vivo. A. Photographs of tumors that developed in xenografttransplanted nude mouse tumor models 5 weeks after injection of DBH-AS1-overexpressing or control SMMC-7721 cells. B. In vivo subcutaneous tumor growth curves were shown for SMMC-7721 cells of Lv-DBH-AS1 and Lv-control vectors. Images C. and weights D. of xenografts established by subcutaneous transplantation with Lv-DBH-AS1-overexpressing and Lv-control SMMC-7721 cells 5 weeks after cell injection. E. H\&E-stained paraffin-embedded sections obtained from xenografts. IHC staining shows that the expression of Ki67 was enhanced in the Lv-DBH-AS1 group compared to the Lv-control group. The higher magnification for the selected region in each part was shown in the right of each part. Original magnification $400 \times$. 

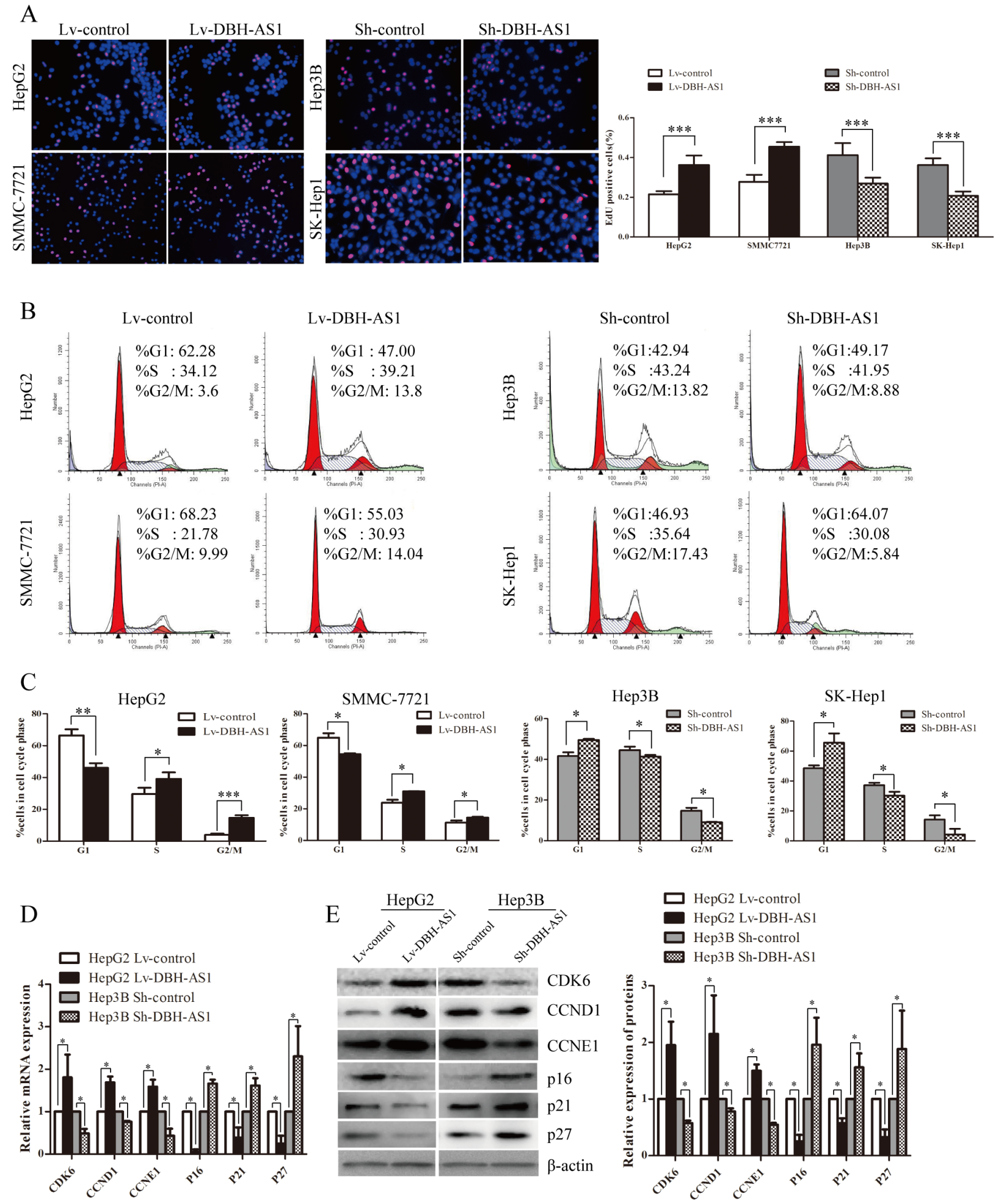

Figure 3: LncRNA DBH-AS1 induces cell-cycle progression in HCC cells. A. HepG2 and SMMC-7721 cells with elevated DBH-AS1 expression were seeded on 96-well plates, and cell proliferation was examined by EdU immunofluorescence staining. Effect of DBH-AS1 knockdown on Hep3B and SK-Hep1 cell proliferation was also measured by EdU immunofluorescence staining. The graph on the right shows the percentage of EdU-positive nuclei. B. Cell-cycle analysis of HepG2 and SMMC-7721 cells overexpressing DBHAS1 and Hep3B and SK-Hep1 cells with stably silenced DBH-AS1 expression. C. Proportion of cells in various phases of the cell cycle. D.-E. The relative expression levels of cell cycle associated genes, including CDK6, CCND1, CCNE1, P16, P21 and P27, were detected in HepG2 cells overexpressing DBH-AS1 and Hep3B cells with stably down-regulated DBH-AS1 expression by qRT-PCR D. and western blot with quantitative analysis $\mathbf{E}$. The results show the means \pm SD from at least 3 separate experiments. ${ }^{*} P<0.05, * * P<0.01,{ }^{* * *} P<$ 0.001 . 
c-Jun N-terminal kinase (JNK) and p38, are crucial molecules involved in pathways associated with cancer pathogenesis.[20] Activation of MAPK signaling pathways in HepG2 cells overexpressing DBH-AS1 was first determined by western blot analysis. Higher levels of phospho-ERK (p-ERK), phospho-p38 (p-p38) and phosphor-JNK (p-JNK) MAPK were observed in DBHAS1 overexpressing HepG2 cells than in control cells (Figure 5A). By contrast, inhibition of DBH-AS1 in Hep3B cells strongly reduced the levels of p-ERK, p-p38 and p-JNK MAPK (Figure 5B).

\section{LncRNA DBH-AS1 is significantly induced by HBx}

Since clinical data showed that the expression of DBH-AS1 was positively correlated with $\mathrm{HBsAg}$, it is possible that $\mathrm{HBV}$ infection would influence the expression of DBH-AS1. Thus, we constructed cell lines that stably re-expressing $\mathrm{HBx}$ by lentivirus infection of hepatic immortal cell line LO2 cells and HCC cell line HepG2 cells (Figure 6A). We noticed that DBH-AS1 was significantly up-regulated in cells stably re-expressing $\mathrm{HBx}$ in comparison with the control group (Figure $6 \mathrm{~B})$. Importantly, levels of DBH-AS1 were higher in HCC patients with HBV infection (31 cases) than those without $\mathrm{HBV}$ infection (14 cases) $(P=0.008$, Figure $6 \mathrm{C})$. Furthermore, to confirm the regulation of DBH-AS1 by HBx in human HCC tissues, we measured DBH-AS1 transcript levels and $\mathrm{HBx}$ mRNA levels in the same set of 31 HCC tissues with HBV infection by qRT-PCR. The results showed that DBH-AS1 transcript level was positively correlated with HBx mRNA level $(R=0.443, P$ $=0.012$, Figure 6D).

\section{LncRNA DBH-AS1 is inactivated by p53}

To investigate the regulator upstream of DBH-AS1, the online JASPAR database (http://jaspar.genereg.net/) was used to analyze the 2000-bp sequence upstream of DBH-AS1 gene. A putative p53-binding site spanned the -447 to $-461 \mathrm{bp}$ positions (Figure 7A). Thus, we addressed whether DBH-AS1 is mediated by P53. The expression of P53 in HepG2 and LO2 cells was down-regulated
A

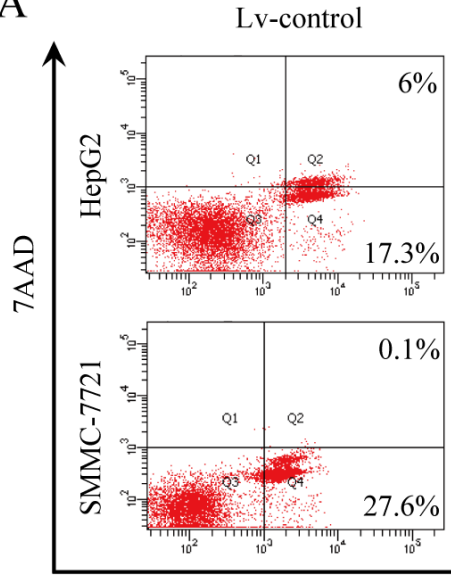

Lv-DBH-AS1

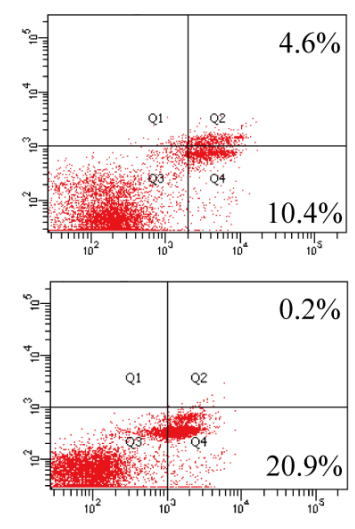

Sh-control

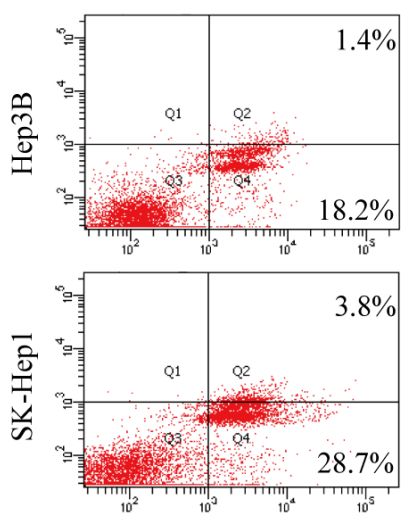

Sh-DBH-AS1

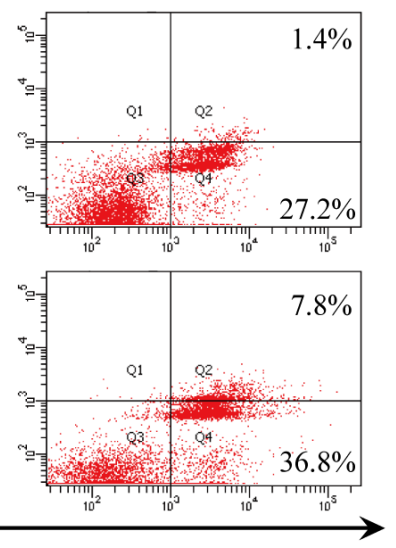

Annexin V-APC

B

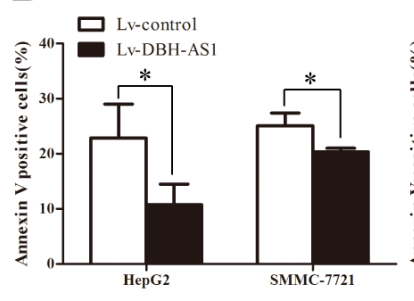

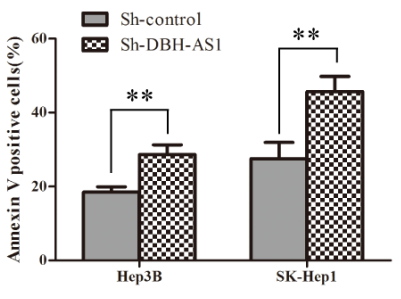

C

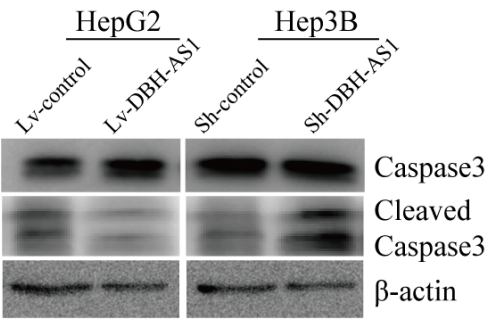

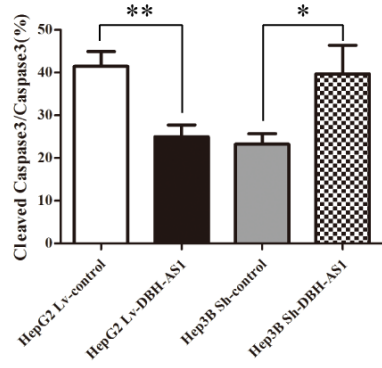

Figure 4: LncRNA DBH-AS1 inhibits serum starvation-induced apoptosis of HCC cells. A. Cells were cultured in serumfree medium for $48 \mathrm{~h}$. The apoptosis rate was measured by FACS-based Annexin-V/7AAD double staining. Cells positive for annexin V staining were counted as apoptotic cells. B. The bar graph shows the percentage of apoptotic cells. C. The levels of Caspase3 and cleaved Caspase 3 were detected by western blot with quantitative analysis in HepG2 cells with elevated DBH-AS1 expression and Hep3B cells with silenced DBH-AS1 expression. The experiments were performed in triplicate; the data are expressed as the mean $\pm \mathrm{SD}$. ${ }^{*} P<0.05$, $* * P<0.01, * * * P<0.001$. 
by transfection with siRNAs targeting the TP53 gene. SiRNAs significantly decreased p53 mRNA and protein levels in HepG2 cells and LO2 cells (Figure 7B and 7C). Meanwhile, DBH-AS1 levels were significantly elevated in cells transfected with anti-TP53 siRNAs compared to those transfected with a scramble control (Figure 7D). Additionally, it is reported that IncRNA H19 can be regulated by p53 under hypoxia.[21] Interestingly, we also observed that DBH-AS1 was up-regulated under hypoxic stress (Figure S1B and S1C).

\section{DISCUSSION}

$\mathrm{HCC}$ is one of the most common cancers worldwide with high prevalence and lethality, which is positively associated with HBV infections [22]. HBx protein encoded by HBV $x$ gene has been elucidated to induce HCC by promoting cell cycle progression and inhibiting the expression of various tumour suppressor genes [6]. Recent studies have indicated that some lncRNAs related to $\mathrm{HBx}$ may act as oncogenes or tumor suppressors, thus involved in HCC pathogenesis $[12,13]$. Although dysregulation of several HBx-related lncRNAs associated with HCC have been identified, the function and clinical significance of the majority of HBx-related lncRNAs in the progression and aggressiveness of $\mathrm{HCC}$ remain unknown.

In this study, we identified a novel HBx-related lncRNA DBH-AS1. Clinical data indicated that high levels of DBH-AS1 were positively correlated with HBsAg and tumor size in HCC patients. Here, we firstly investigated the biological functions of DBH-AS1 by gain-of-function and loss-of-function experiments. We provided evidence that DBH-AS1 promoted $\mathrm{HCC}$ cell proliferation in vitro and in vivo. Mechanistically, DBH-AS1 was shown to induce cell cycle progression by accelerating G1/S and $\mathrm{G} 2 / \mathrm{M}$ transition. It has been well-defined that tumourassociated cell cycle defects are often mediated by the accumulation of cyclins (CCNs) and cyclin-dependent kinases (CDKs) complexes and reduced activity of cyclindependent kinase inhibitors (CDKIs)[23,24]. Our data suggest that DBH-AS1 can modulate several cell-cycle related factors, including down-regulating p16, p21 and p27 (members of CDKIs) as well as up-regulating CDK6 (a member of CDKs), CCND1, CCNE1 (members of $\mathrm{CCNs}$ ). In addition to enhanced proliferation, resistance to apoptosis is also a hallmark of cancer cells [25]. In this study we also found that DBH-AS1 can protect HCC cells from serum starvation-induced apoptosis and promote

A
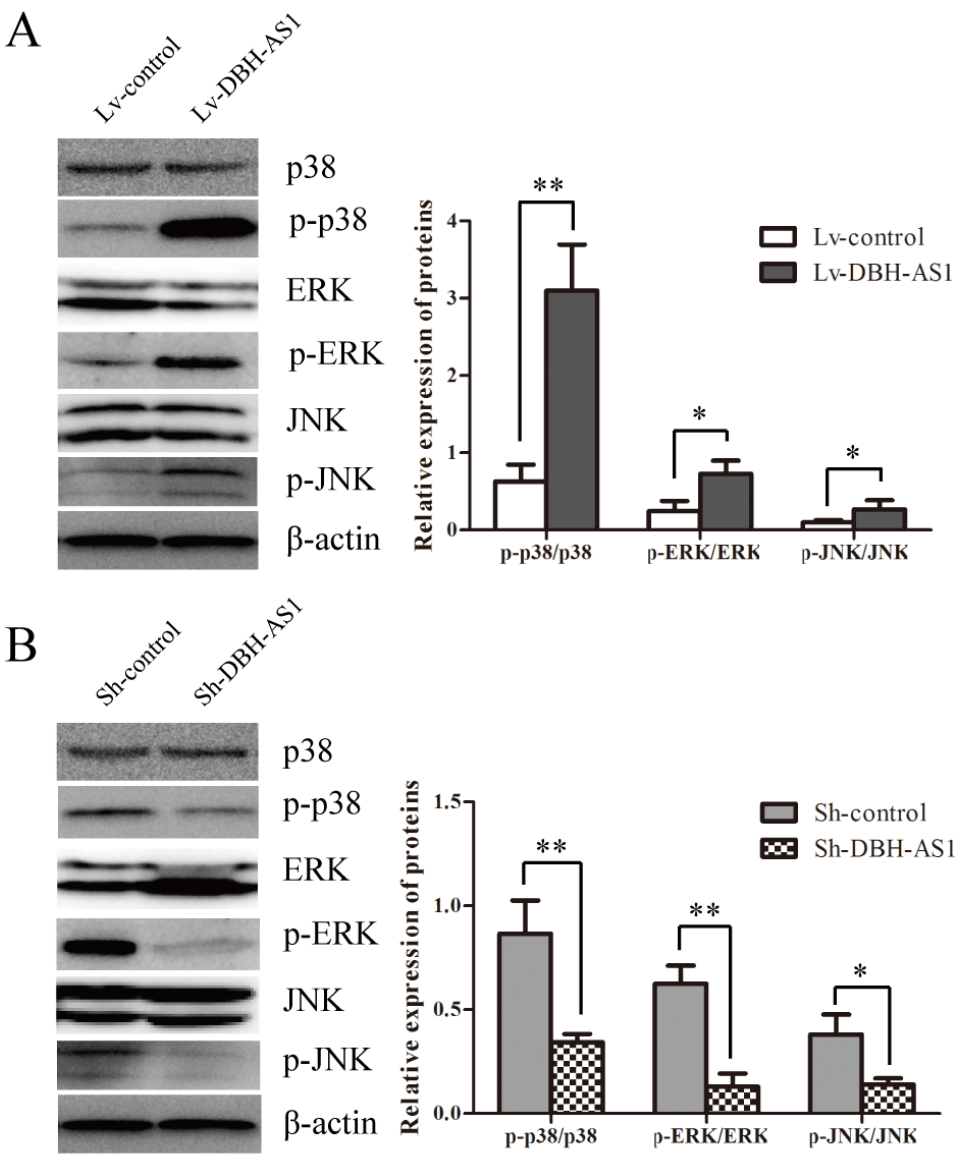

Figure 5: LncRNA DBH-AS1 activates MAPK signaling pathways. The levels of ERK, p38, JNK, p-ERK, p-p38 and p-JNK were examined by western blot analysis in HepG2 cells overexpressing DBH-AS1 A. and Hep3B cells with silenced DBH-AS1 expression B. The experiments were performed in triplicate; the data are expressed as the mean $\pm \mathrm{SD}$. $* P<0.05,{ }^{*} P<0.01, * * * P<0.001$. 
A

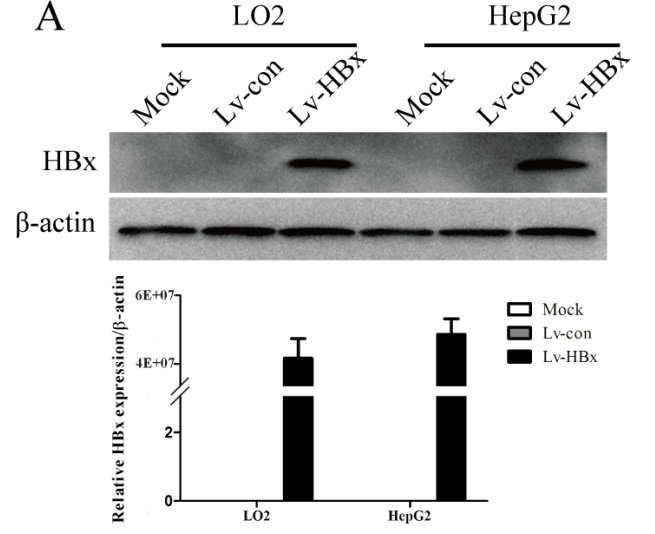

$\mathrm{C}$

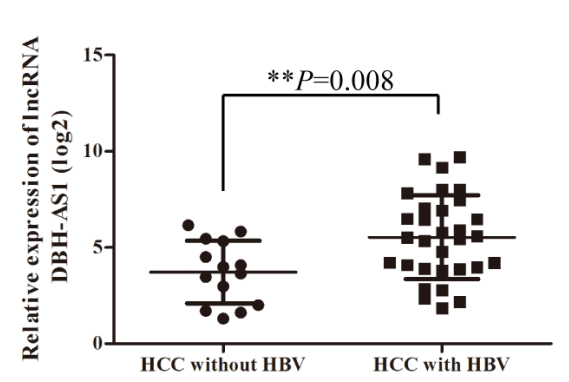

B

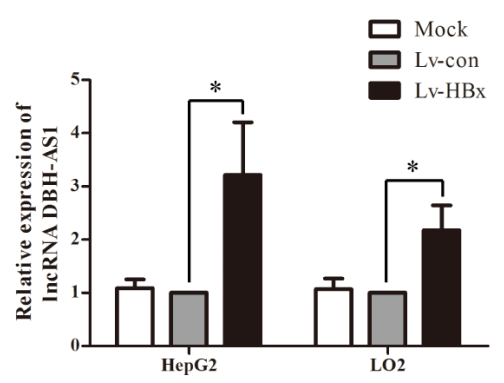

$\mathrm{D}$

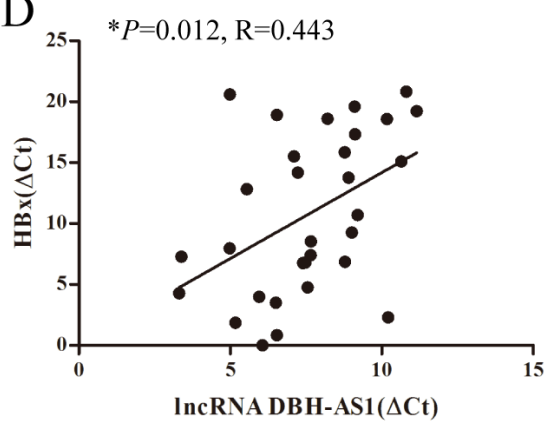

Figure 6: HBx induces the expression of IncRNA DBH-AS1. A. Ectopic re-expression of HBx was detected in Lv-HBx-transfected HepG2 and LO2 cells by qRT-PCR and western blot. $\beta$-actin was used as a loading control. $\mathbf{B}$. The relative expression of lncRNA DBHAS1 in HepG2 and LO2 cells re-expressing HBx compared with controls by qRT-PCR. Data are shown as the mean \pm SD based on at least three independent experiments. C. Comparison of levels of DBH-AS1 in HCC patients with and without HBV infection (independent $t$ test). D. The correlation between DBH-AS1 transcript level and HBx mRNA level in $31 \mathrm{HCC}$ tissues. The $\Delta \mathrm{Ct}$ values were subjected to Pearson correlation analysis. $* P<0.05, * * P<0.01, * * * P<0.001$.

A

\begin{tabular}{|c|c|c|c|c|c|c|c|}
\hline Model ID & Model name & Score & Relative score & Start & End & Strand & Predicted site sequence \\
\hline MA0106.2 & TP53 & 7.715 & 0.807965711 & 447 & 461 & -1 & GCAGGACCAGGCATT \\
\hline
\end{tabular}

B

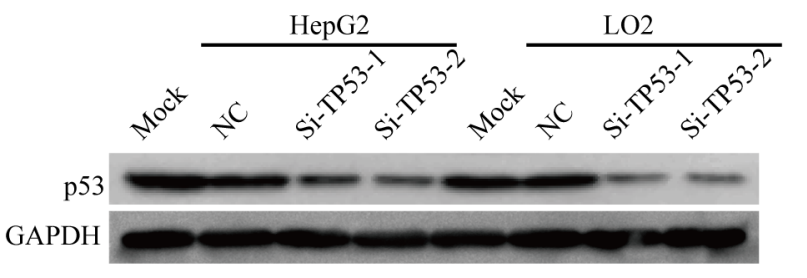

$\mathrm{D}$

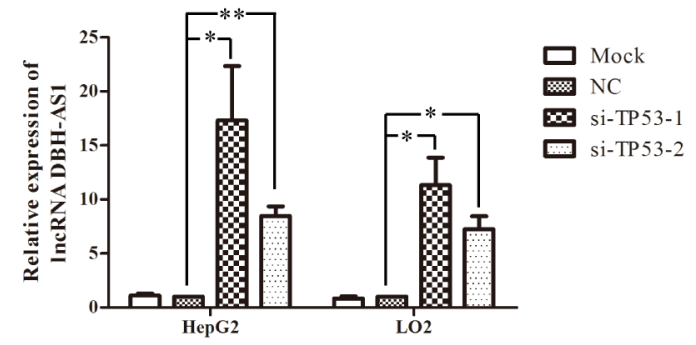

$\mathrm{C}$

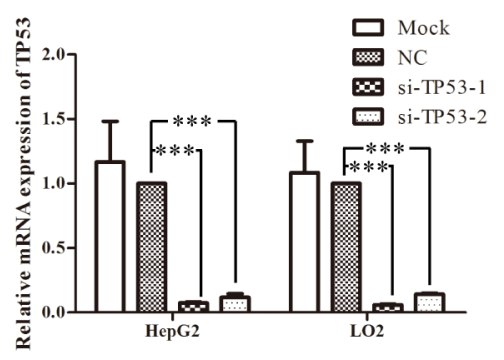

Figure 7: LncRNA DBH-AS1 is inactivated by p53. A. The potential p53-binding site upstream of DBH-AS1 predicted by JASPAR database. B. Western blot analysis showed the reduced levels of p53 protein in HepG2 cells and LO2 cells transfected with siRNAs. C. Reduced p53 mRNA expression by siRNAs in HepG2 cells and LO2 cells was shown by qRT-PCR. D. Expression of DBH-AS1 transcripts was quantified by qRT-PCR. Data shown are the mean \pm SD of three independent experiments. ${ }^{*} P<0.05,{ }^{*} P<0.01,{ }^{* * *} P<0.001$. 
cell survival. This is the first report to demonstrate the functional significance of DBH-AS1 in HCC, and our results indicate that DBH-AS1 may function as an oncogene. Consistent with our results, Magkoufopoulou, C. et al [14] reported that the anti-cancer drug quercetin can reduce the expression of DBH-AS1 in HepG2 cells, partly in support to our hypothesis.

Currently, ERK/p38/JNK MAPK signaling pathways have been demonstrated to regulate a variety of cellular activities, including proliferation, differentiation, survival, and death $[20,26]$. ERK/p38/JNK MAPK signaling can not only regulate cell cycle progression at different transition points but also modulate the cellular programmes for cell survival and differentiation[26,27] . Once activated, ERK/p38/JNK MAPK could induce the levels of CDK6, CCND1 and CCNE1, and reduce the levels of p16, p21 and p27, thereby inducing G1/s and $\mathrm{G} 2 / \mathrm{M}$ transition and cell proliferation [28-30]. Additionally, some studies reported that activation of ERK/p38/JNK MAPK resists apoptosis and promotes cell survival in HCC [27]. Here, we observed higher levels of p-ERK, p-p38 and p-JNK in HepG2 cells overexpressing DBH-AS1 as well as lower levels of p-EKR, p-p38 and p-JNK in Sh-DBH-AS1 Hep3B cells than control group separately. Our data suggests that ERK/p38/JNK MAPK signaling pathway can be activated by DBH-AS1 and may be partially responsible for DBH-AS1-induced cell proliferation and survival.

Given the results we obtained in clinical data analysis that the expression of DBH-AS1 is correlated to HBsAg, we wonder whether DBH-AS1 could be regulated by $\mathrm{HBx}$ protein. $\mathrm{HBx}$ has been demonstrated to promote liver cell proliferation and inhibit cell apoptosis via MAPK signaling $[31,32]$. Therefore, we focused on the investigation of the relationship between $\mathrm{HBx}$ and DBH-AS1. Our findings confirmed that DBH-AS1 can be markedly induced by $\mathrm{HBx}$ protein in hepatocytes. Moreover, clinical data indicated that higher levels of DBH-AS1 were found in HCC patients with $\mathrm{HBV}$ infection than those without HBV infection.

Increasing evidence has shown that the transcript factor p53 plays an important role in cell cycle arrest, senescence and apoptosis, thus suppressing the development of various cancers [33]. Recent studies have characterized a series of p53-related lncRNAs, such as lincRNA-p21[34], GAS5 [35], MEG3 [36], PVT1 [37], loc285194 [38]. Importantly, HBx protein has been reported to bind to and inhibit the expression of p53 and other tumour suppressor genes and senescencerelated factors $[39,40]$. Thus, we wonder whether there is a relationship between p53 and HBx-related lncRNA DBH-AS1 in HCC. Interestingly, we found a putative p53-binding site among the 2000-bp sequence upstream of DBH-AS1 gene and suppressed p53 expression resulted in elevated expression of DBH-AS1. But whether p53 can directly bind to the promoter region of DBH-AS1 still remains further investigated. We will next identify the promoter region of DBH-AS1 gene and use chromatin immunoprecipitation assays to further confirm the directly interaction between p53 and DBH-AS1 gene. Additionally, consistent with the finding reported by Matouk, I. J. [21] that a functional link exists between p53, hypoxia and lncRNA H19, we observed upregulation of DBH-AS1 under hypoxia stress, indicating that there might also be a link between p53 and DBH-AS1 under hypoxic stress.

Although we confirm that HBx-related lncRNA DBH-AS1 may act as an oncogene to promote cell proliferation and survival via MAPK signaling pathway, the molecules directly functioning downstream of DBHAS1 still remain unclear. Consequently, further studies such as RNA-binding protein immunoprecipitation assays are necessary to find out the proteins or microRNAs directly binding with DBH-AS1. Additionally, it is of great necessity for us to collect adjacent HCC tissues or normal liver tissues and then compare the expression of $\mathrm{DBH}$ AS1 between HCC tumor tissues and adjacent tissues or normal liver tissues.

In summary, our findings suggest that lncRNA DBHAS1 promotes cell proliferation and survival via MAPK signaling in HCC. Modulation of the tumor proliferation effect through inhibiting MAPK activation mediated by DBH-AS1 overexpression might be used as a potential target for HCC prevention and therapy. Moreover, DBHAS1 is found to be induced by HBx and inactivated by p53.We primarily confirm the regulatory mechanism of DBH-AS1 in HCC progression. Our findings demonstrate that $\mathrm{DBH}-\mathrm{AS} 1$ is a potential oncogene participating in $\mathrm{HCC}$ pathogenesis.

\section{MATERIALS AND METHODS}

\section{Cell lines and patient samples}

The HepG2, SMMC-7721, Hep3B, MHCC97H, SK-Hep1 human HCC cell lines and the LO2, QSG7701 human immortalized normal hepatocytes were obtained from the Cell Bank of Type Culture Collection (Chinese Academy of Sciences, Shanghai, China). Cells were cultured in Dulbecco's modified Eagle's medium (DMEM, Gibco, Gaithersburg, MD, USA) containing 10\% fetal bovine serum (FBS, Gibco) and incubated at $37^{\circ} \mathrm{C}$ in an atmosphere of 5\% CO2. HCC tissues were obtained from patients who had undergone routine surgery from 2012 to 2014 at Nanfang Hospital, Southern Medical University. All tissues were histopathologically confirmed as HCC. Written informed consent for the biological studies was obtained from each patient involved in the study, and the study was approved by the Ethics Committee of Nanfang Hospital. 


\section{RNA extraction and real-time quantitative PCR analysis (qRT-PCR)}

Total RNA was extracted from cultured cells or tissues using TRIzol Reagent (Takara, Dalian, China). For IncRNA DBH-AS1, first-strand cDNA was synthesized using the M-MLV Reverse Transcriptase (Promega, Madison WI, USA). For mRNAs, cDNA was generated using the PrimeScript RT reagent kit (Takara). The RNA expression levels were measured by qRT-PCR using SYBR Green PCR Master Mix (Takara) which was performed on the ABI 7500 Fast Real Time PCR system (Applied Biosystems, Foster City, CA, USA). U6 and $\beta$-actin were used as internal controls. All results were expressed as the means $\pm \mathrm{SD}$ of at least three independent experiments. Comparative quantification was determined using the $2^{-\Delta \Delta \mathrm{Ct}}$ method. The primers used are presented in Supplementary Table S1.

\section{Western blot analysis}

Total proteins were prepared from the samples by complete cell lysis (Keygen Biotech, Jiangsu, China) with protease and phosphatase inhibitors. Identical quantities of proteins were separated on sodium dodecyl sulfatepolyacrylamide gel electrophoresis gels and transferred onto polyvinylidene difluoride membranes. After incubation with antibodies specific for HBx (Abcam, Cambridge, UK), CDK6 (Cell Signaling Technology, Beverly, MA, USA), CCND1(Cell Signaling Technology ), CCNE1(Cell Signaling Technology), p16(Cell Signaling Technology ), p21(Cell Signaling Technology), p27(Cell Signaling Technology), Caspase3(Immunoway, USA), cleaved Caspase3(Cell Signaling Technology), ERK (Cell Signaling Technology), p-ERK (Cell Signaling Technology), p38 (Cell Signaling Technology), p-p38 (Cell Signaling Technology), JNK(Cell Signaling Technology), p-JNK(Cell Signaling Technology), p53(Millipore, Schwalbach/Ts., Germany), HIF-1 $\alpha$ (Abcam), GAPDH (Cell Signaling Technology) and $\beta$-actin (Proteintech, USA), the blots were incubated with goat anti-rabbit or anti-mouse secondary antibodies (Bioss, Beijing, China). The proteins were visualized using a chemiluminescence method (ECL Plus Western Blotting Detection System; Amersham Biosciences, Foster City, CA, USA) and quantified by ImageJ software.

\section{Construction of stable cell lines}

To obtain cell lines stably overexpressing DBHAS1, HepG2 and SMMC7721 cells were infected with the Lv-DBH-AS1 and Lv-control viruses (GeneChem, Shanghai, China). To observe the knockdown effects of DBH-AS1 in vitro, Hep3B and SK-Hep1 cells were transfected with the shRNA-DBH-AS1 (Sh-DBH-AS1) or control (Sh-con) viruses (GeneChem). Recombinant lentiviruses containing HBx (Lv-HBx) or control (Lv-con) purchased from LAND (Guangzhou, China) were used to infect HepG2 and LO2 cell lines. The infection efficiency was confirmed by qRT-PCR or western blot.

\section{Cell counting kit-8 (CCK-8) assay}

The cell viability was assessed by CCK8 (Dojindo Laboratories, Kumamoto, Japan) according to the manufacturer's protocol. All of the experiments were performed in sixth. The cell proliferation curves were plotted using the absorbance at each time point.

\section{5-ethynyl-2'-deoxyuridine (EdU) incorporation assay}

EdU incorporation assay was carried out using the Cell-Light EdU imaging detecting kit (RiboBio, Guangzhou, China) according to the manufacturer's instructions. Briefly, cells were seeded in 96-well plates at a density of 5000 cells/well. After adherence, we added EdU labeling medium and incubated for about $60 \mathrm{~min}$. After fixed with $4 \%$ formaldehyde for 15 min and treated with $0.5 \%$ Triton X-100 for $20 \mathrm{~min}$, cells were exposed to Apollo reaction cocktail for $30 \mathrm{~min}$. Subsequently, the DNA from cells in each well was stained with Hoechst $33342(5 \mathrm{ug} / \mathrm{mL})$ for $30 \mathrm{~min}$ and visualized under a fluorescent microscope.

\section{Colony formation assay}

Cells were seeded in 6-well plates at a density of 100cells/well. After incubation for 14 days, cells were washed twice with PBS, fixed with methanol and stained with crystal violet. The number of colonies containing > 50cells was counted under a microscope.

\section{Cell cycle analysis}

Cells were harvested and washed twice with PBS. After fixed in $70 \%$ ice-cold ethanol and kept overnight at $4^{\circ} \mathrm{C}$, cells were stained with propidium iodide supplemented with RNaseA (Keygen Biotech) for $30 \mathrm{~min}$ at $37^{\circ} \mathrm{C}$. The DNA content of labeled cells was acquired using FACS cytometry (BD Biosciences Inc., Franklin Lakes, NJ, USA). Each experiment was performed in triplicate. 


\section{Cell apoptosis analysis}

Cells were cultured in serum-free medium for $48 \mathrm{~h}$. Then, the apoptosis assay was done with the Annexin V-7AAD apoptosis detection kit (Keygen Biotech) according to the manufacturer's instructions. Cells were then analyzed by FACS cytometry (BD Biosciences Inc.).

\section{In vivo tumor growth assay}

All investigations were approved by the Animal Experimental Committee of Nanfang Hospital. The male $\mathrm{BALB} / \mathrm{C}$ nude mice were purchased from the Guangdong Experimental Animal Center of the Chinese Academy of Sciences, bred and maintained in a specific pathogen-free facility. For xenograft models: cells were collected by trypsinization, washed twice with PBS, and resuspended with serum-free medium. A total of $5 \times 10^{6}$ SMMC-7721 cells stably transfected with Lv-DBH-AS1 or Lv-control in $0.1 \mathrm{ml}$ DMEM medium was independently injected subcutaneously into the left back and right back of 9 nude mice. Tumor size was monitored routinely for 5 weeks. Mean percent of body weight ( \pm SEM) and tumor size for each group was measured every 7 days.

\section{Immunohistochemistry}

Immunohistochemistry for Ki-67 was performed on paraffin sections using a primary antibody against Ki67 (Abcam) and a horseradish peroxidase-conjugated rabbit anti-goat antibody (Maixin, Fuzhou, China), and the proteins in situ were visualized with 3, 3-diaminobenzidine and analyzed using a bright field microscope.

\section{RNA interference and transfection}

HepG2 and LO2 cells were plated in a 6-well plate at a $30-50 \%$ confluence. After $24 \mathrm{~h}$, siRNA against TP53 (Ambion, Carlsbad, CA) were transfected into HepG2 and LO2 cells using Lipofectamine 2000 reagent (Invitrogen, USA) according to the manufacturer's instructions. Cells transfected with the transfection agent, but no siRNA (Mock) or scramble-control siRNA (NC) were used as controls. The cells were harvested 48 hours after transfection.

\section{Statistical analysis}

SPSS 13.0 software (SPSS Inc., Chicago, IL, USA) and GraphPad software (GraphPad Software, Inc., La Jolla, CA, USA) were used to analyze all data for statistical significance. The Chi-Square test was applied to the examination of relationship between IncRNA DBH-AS1 expression levels and clinicopathological characteristics. Two-tailed Student's $t$-test was used for comparisons of two independent groups. One-way ANOVA was used to determine the differences between groups for all in vitro analyses. Statistical significance was set at $* P<0.05, * * P$ $<0.01,{ }^{* * *} P<0.001 . P<0.05$ was considered statistically significant.

\section{Abbreviations}

HCC: hepatocellular carcinoma; HBV: hepatitis B virus; $\mathrm{HBx}$ : hepatitis $\mathrm{B}$ virus x protein; lncRNA: long noncoding RNA; HBsAg: hepatitis B surface antigen; qRTPCR: quantitative real-time polymerase chain reaction; FACS: fluorescence-activated cell sorting; siRNA: small interfering RNA; MAPK: mitogen-activated protein kinase; ERK: extracellular signal-regulated kinase; JNK: c-Jun N-terminal kinase.

\section{ACKNOWLEDGMENTS}

We thank Department of Hepatobiliary Surgery, Nanfang Hospital, Southern Medical University, Guangzhou, China for providing the HCC tissue samples and related anonymous clinical data. This work was supported by grants from National Natural Science Foundation of China (81271932) and Doctoral Program of the Ministry of Education (20134433110010).

\section{CONFLICTS OF INTEREST}

The authors declare no conflict of interest.

\section{REFERENCES}

1. Cervello, M., McCubrey, J.A., Cusimano, A., Lampiasi, N., Azzolina, A. and Montalto, G. Targeted therapy for hepatocellular carcinoma: novel agents on the horizon. Oncotarget.2012; 3: 236-60.

2. El-Serag, H.B. Hepatocellular carcinoma. N Engl J Med.2011; 365: 1118-27.

3. Arzumanyan, A., Reis, H.M. and Feitelson, M.A. Pathogenic mechanisms in HBV- and HCV-associated hepatocellular carcinoma. Nat Rev Cancer.2013; 13: 12335.

4. Villanueva, A., Hernandez-Gea, V. and Llovet, J.M. Medical therapies for hepatocellular carcinoma: a critical view of the evidence. Nat Rev Gastroenterol Hepatol.2013; 10: 34-42.

5. de Lope, C.R., Tremosini, S., Forner, A., Reig, M. and Bruix, J. Management of HCC. J Hepatol.2012; 56 Suppl 1: S75-87.

6. Kew, M.C. Hepatitis B virus x protein in the pathogenesis of hepatitis B virus-induced hepatocellular carcinoma. J 
Gastroenterol Hepatol.2011; 26 Suppl 1: 144-52.

7. Wang, K.C. and Chang, H.Y. Molecular mechanisms of long noncoding RNAs. Mol Cell.2011; 43: 904-14.

8. Huang, J.L., Zheng, L., Hu, Y.W. and Wang, Q. Characteristics of long non-coding RNA and its relation to hepatocellular carcinoma. Carcinogenesis.2014; 35: 507-14.

9. Yuan, J.H., Yang, F., Wang, F., Ma, J.Z., Guo, Y.J., Tao, Q.F., Liu, F., Pan, W., Wang, T.T., Zhou, C.C., Wang, S.B., Wang, Y.Z. and Yang, Y.et al. A long noncoding RNA activated by TGF-beta promotes the invasion-metastasis cascade in hepatocellular carcinoma. Cancer Cell.2014; 25: 666-81.

10. Xu, W.H., Zhang, J.B., Dang, Z., Li, X., Zhou, T., Liu, J., Wang, D.S., Song, W.J. and Dou, K.F. Long non-coding RNA URHC regulates cell proliferation and apoptosis via ZAK through the ERK/MAPK signaling pathway in hepatocellular carcinoma. Int J Biol Sci.2014; 10: 664-76.

11. Cao, C., Sun, J., Zhang, D., Guo, X., Xie, L., Li, X., Wu, D. and Liu, L. The Long Intergenic Noncoding RNA UFC1, a Target of MicroRNA 34a, Interacts With the mRNA Stabilizing Protein HuR to Increase Levels of beta-Catenin in HCC Cells. Gastroenterology.2015; 148: 415-426.e18.

12. Huang, J.F., Guo, Y.J., Zhao, C.X., Yuan, S.X., Wang, Y., Tang, G.N., Zhou, W.P. and Sun, S.H. Hepatitis B virus $\mathrm{X}$ protein (HBx)-related long noncoding RNA (lncRNA) down-regulated expression by $\mathrm{HBx}$ (Dreh) inhibits hepatocellular carcinoma metastasis by targeting the intermediate filament protein vimentin. Hepatology.2013; 57: 1882-92.

13. Du Y, Kong, G., You, X., Zhang, S., Zhang, T., Gao, Y., Ye, L. and Zhang, X. Elevation of highly up-regulated in liver cancer (HULC) by hepatitis B virus X protein promotes hepatoma cell proliferation via down-regulating p18. J Biol Chem.2012; 287: 26302-11.

14. Magkoufopoulou, C., Claessen, S.M., Jennen, D.G., Kleinjans, J.C. and van Delft, J.H. Comparison of phenotypic and transcriptomic effects of false-positive genotoxins, true genotoxins and non-genotoxins using HepG2 cells. Mutagenesis.2011; 26: 593-604.

15. Gilbert, J.R., Kumar, A., Newey, S., Rao, N., Ioannou, P., Qiu, H., Lin, D., Xu, P., Pettenati, M.J. and Pericak-Vance, M.A. Physical and cDNA mapping in the DBH region of human chromosome 9q34. Hum Hered.2000; 50: 151-7.

16. Russo, M., Spagnuolo, C., Tedesco, I., Bilotto, S. and Russo, G.L. The flavonoid quercetin in disease prevention and therapy: facts and fancies. Biochem Pharmacol.2012; 83: 6-15.

17. Sudan, S. and Rupasinghe, H.P. Quercetin-3-O-glucoside induces human DNA topoisomerase II inhibition, cell cycle arrest and apoptosis in hepatocellular carcinoma cells. Anticancer Res.2014; 34: 1691-9.

18. Zhao, J.L., Zhao, J. and Jiao, H.J. Synergistic growthsuppressive effects of quercetin and cisplatin on HepG2 human hepatocellular carcinoma cells. Appl Biochem
Biotechnol.2014; 172: 784-91.

19. Hsieh, A., Kim, H.S., Lim, S.O., Yu, D.Y. and Jung, G. Hepatitis B viral X protein interacts with tumor suppressor adenomatous polyposis coli to activate Wnt/beta-catenin signaling. Cancer Lett.2011; 300: 162-72.

20. Kim, E.K. and Choi, E.J. Pathological roles of MAPK signaling pathways in human diseases. Biochim Biophys Acta.2010; 1802: 396-405.

21. Matouk, I.J., Mezan, S., Mizrahi, A., Ohana, P., Abu-Lail, R., Fellig, Y., Degroot, N., Galun, E. and Hochberg, A. The oncofetal H19 RNA connection: hypoxia, p53 and cancer. Biochim Biophys Acta.2010; 1803: 443-51.

22. El-Serag, H.B. Epidemiology of viral hepatitis and hepatocellular carcinoma. Gastroenterology.2012; 142: 1264-1273.e1.

23. Bonelli, P., Tuccillo, F.M., Borrelli, A., Schiattarella, A. and Buonaguro, F.M. CDK/CCN and CDKI alterations for cancer prognosis and therapeutic predictivity. Biomed Res Int.2014; 2014: 361020.

24. Malumbres, M. and Barbacid, M. Cell cycle, CDKs and cancer: a changing paradigm. Nat Rev Cancer.2009; 9: 15366.

25. Evan, G.I. and Vousden, K.H. Proliferation, cell cycle and apoptosis in cancer. Nature.2001; 411: 342-8.

26. Wagner, E.F. and Nebreda, A.R. Signal integration by JNK and p38 MAPK pathways in cancer development. Nat Rev Cancer.2009; 9: 537-49.

27. Min, L., He, B. and Hui, L. Mitogen-activated protein kinases in hepatocellular carcinoma development. Semin Cancer Biol.2011; 21: 10-20.

28. Meloche, S. and Pouyssegur, J. The ERK1/2 mitogenactivated protein kinase pathway as a master regulator of the G1- to S-phase transition. Oncogene.2007; 26: 3227-39.

29. Liu, D., Liu, J., Lin, B., Liu, S., Hou, R., Hao, Y., Liu, Q., Zhang, S. and Iwamori, M. Lewis y regulate cell cycle related factors in ovarian carcinoma cell RMG-I in vitro via ERK and Akt signaling pathways. Int J Mol Sci.2012; 13: 828-39.

30. Sherr, C.J. and Roberts, J.M. CDK inhibitors: positive and negative regulators of G1-phase progression. Genes Dev.1999; 13: 1501-12.

31. Shan, C., Xu, F., Zhang, S., You, J., You, X., Qiu, L., Zheng, J., Ye, L. and Zhang, X. Hepatitis B virus X protein promotes liver cell proliferation via a positive cascade loop involving arachidonic acid metabolism and p-ERK1/2. Cell Res.2010; 20: 563-75.

32. Arbuthnot, P., Capovilla, A. and Kew, M. Putative role of hepatitis B virus X protein in hepatocarcinogenesis: effects on apoptosis, DNA repair, mitogen-activated protein kinase and JAK/STAT pathways. J Gastroenterol Hepatol.2000; 15: 357-68.

33. Reinhardt, H.C. and Schumacher, B. The p53 network: cellular and systemic DNA damage responses in aging and cancer. Trends Genet.2012; 28: 128-36. 
34. Huarte, M., Guttman, M., Feldser, D., Garber, M., Koziol, M.J., Kenzelmann-Broz, D., Khalil, A.M., Zuk, O., Amit, I., Rabani, M., Attardi, L.D., Regev, A. and Lander, E.S.et al. A large intergenic noncoding RNA induced by p53 mediates global gene repression in the p53 response. Cell.2010; 142: 409-19.

35. Krell, J., Frampton, A.E., Mirnezami, R., Harding, V., De Giorgio, A., Roca, A.L., Cohen, P., Ottaviani, S., Colombo, T., Jacob, J., Pellegrino, L., Buchanan, G. and Stebbing, J.et al. Growth arrest-specific transcript 5 associated snoRNA levels are related to p53 expression and DNA damage in colorectal cancer. PLoS One.2014; 9: e98561.

36. Lu, K.H., Li, W., Liu, X.H., Sun, M., Zhang, M.L., Wu, W.Q., Xie, W.P. and Hou, Y.Y. Long non-coding RNA MEG3 inhibits NSCLC cells proliferation and induces apoptosis by affecting p53 expression. BMC Cancer.2013; 13: 461.

37. Barsotti, A.M., Beckerman, R., Laptenko, O., Huppi, K., Caplen, N.J. and Prives, C. p53-Dependent induction of PVT1 and miR-1204. J Biol Chem.2012; 287: 2509-19.

38. Liu, Q., Huang, J., Zhou, N., Zhang, Z., Zhang, A., Lu, Z., Wu, F. and Mo, Y.Y. LncRNA loc285194 is a p53regulated tumor suppressor. Nucleic Acids Res.2013; 41: 4976-87.

39. Xu, X., Fan, Z., Kang, L., Han, J., Jiang, C., Zheng, X., Zhu, Z., Jiao, H., Lin, J., Jiang, K., Ding, L., Zhang, H. and Cheng, L.et al. Hepatitis B virus $\mathrm{X}$ protein represses miRNA-148a to enhance tumorigenesis. J Clin Invest.2013; 123: 630-45.

40. Hussain, S.P., Schwank, J., Staib, F., Wang, X.W. and Harris, C.C. TP53 mutations and hepatocellular carcinoma: insights into the etiology and pathogenesis of liver cancer. Oncogene.2007; 26: 2166-76. 